\title{
The impact of closed-loop intracortical stimulation on neural activity in brain- injured, anesthetized animals
}

\author{
Marta Carè ${ }^{1,2 \dagger}$, Alberto Averna ${ }^{1,3+}$, Federico Barban ${ }^{1,2}$, Marianna Semprini ${ }^{1}$, Lorenzo De Michieli ${ }^{1}$, \\ Randolph J. Nudo ${ }^{4,5}$, David J. Guggenmos ${ }^{4, *^{*}}$ and Michela Chiappalone $e^{1,2^{*}+}$ (D)
}

\begin{abstract}
Background: Acquired brain injuries, such as stroke, are a major cause of long-term disability worldwide. Intracortical microstimulation (ICMS) can be used successfully to assist in guiding appropriate connections to restore lost sensorimotor integration. Activity-Dependent Stimulation (ADS) is a specific type of closed-loop ICMS that aims at coupling the activity of two different brain regions by stimulating one in response to activity in the other. Recently, ADS was used to effectively promote behavioral recovery in rodent models following a unilateral traumatic brain injury in the primary motor cortex. While behavioral benefits have been described, the neurophysiological changes in spared areas in response to this type of stimulation have not been fully characterized. Here we explored how single-unit spiking activity is impacted by a focal ischemic lesion and, subsequently, by an ADS treatment.
\end{abstract}

Methods: Intracortical microelectrode arrays were implanted in the ipsilesional rostral forelimb area (RFA) to record spike activity and to trigger intracortical microstimulation in the primary somatosensory area (S1) of anaesthetized Long Evans rats. An ischemic injury was induced in the caudal forelimb area through microinjections of Endothelin1. Activity from both RFA and S1 was recorded and analyzed off-line by evaluating possible changes, either induced by the lesion in the Control group or by stimulation in the ADS group.

Results: We found that the ischemic lesion in the motor area led to an overall increase in spike activity within RFA and a decrease in S1 with respect to the baseline condition. Subsequent treatment with ADS increased the firing rate in both RFA and S1. Post-stimulation spiking activity was significantly higher compared to pre-stimulation activity in the ADS animals versus non-stimulated controls. Moreover, stimulation promoted the generation of highly synchronized bursting patterns in both RFA and S1 only in the ADS group.

Conclusions: This study describes the impact on single-unit activity in ipsilesional areas immediately following a cortical infarct and demonstrates that application of ADS is effective in altering this activity.

Keywords: Activity-dependent stimulation, Firing, In vivo, Micro-electrode arrays, Spike, Stroke, Synchronization

\footnotetext{
* Correspondence: DGUGGENMOS@kumc.edu; michela.chiappalone@unige.it

${ }^{\dagger}$ Marta Carè and Alberto Averna are equal first author.

${ }^{\dagger}$ David J. Guggenmos and Michela Chiappalone are equal last author.

${ }^{4}$ Department of Rehabilitation Medicine, University of Kansas Medical Center,

Kansas City 66160, USA

${ }^{1}$ Rehab Technologies, Istituto Italiano di Tecnologia, 16163 Genoa, Italy

Full list of author information is available at the end of the article
}

(c) The Author(s). 2022 Open Access This article is licensed under a Creative Commons Attribution 4.0 International License, which permits use, sharing, adaptation, distribution and reproduction in any medium or format, as long as you give appropriate credit to the original author(s) and the source, provide a link to the Creative Commons licence, and indicate if changes were made. The images or other third party material in this article are included in the article's Creative Commons licence, unless indicated otherwise in a credit line to the material. If material is not included in the article's Creative Commons licence and your intended use is not permitted by statutory regulation or exceeds the permitted use, you will need to obtain permission directly from the copyright holder. To view a copy of this licence, visit http://creativecommons.org/licenses/by/4.0/. 


\section{Background}

Acquired brain injuries, such as stroke, are a major cause of death and long-term disability worldwide (Organization 2018). When an ischemic stroke occurs, there is a short, hours-long window in which a resolution of the occlusion, either through mechanical or chemical interventions such as thrombectomy or tissue plasminogen activator, is possible (Phipps and Cronin 2020). Reperfusion of brain tissue is critical because, once the neurons are lost, functional and behavioral impairment will occur. If an ischemic injury occurs within the primary motor cortex (M1), as is common in stroke, there is an initial loss of descending information to the spinal cord, which leads to hemiparesis and other motor dysfunctions. In addition, there is a widespread disruption in communication throughout the sensorimotor regions such as the primary somatosensory cortex (S1) and premotor areas that is thought to contribute to the severity of the injury. While there have been investigations into the neural response to ischemic conditions at the site of injury, there is far less information on the immediate neural response of these connected regions in the period immediately following the injury.

Since only a minority of stroke survivors are able to achieve functional independence in simple activities of daily living, promoting the recovery of disabled patients is a primary challenge in scientific and clinical research (Semprini, Laffranchi et al. 2018). The standard-of-care for recovering lost functions following injury is physical therapy, which utilizes neuroplastic mechanisms to promote reorganization of spared regions and ultimately improvements in motor outcomes, but its effects are often limited or incomplete (Dimyan and Cohen 2011, Mang, Campbell et al. 2013, Belagaje 2017, Lang, Waddell et al. 2021). It is clear that there is a window of time after the initial injury in which the brain is more amenable to these neuroplastic mechanisms, starting shortly after ischemia occurs and ebbing at around 3 months (Murphy and Corbett 2009, Belagaje 2017, Coleman, Moudgal et al. 2017). Given the loss of the tissue in the ischemic core, it is likely that novel treatments will focus on the restoration of function through the spared, formerly connected areas to these regions.

To this end, we developed a treatment that reconnects brain regions that have become disconnected as a result of an acquired brain injury. Our strategy, activitydependent stimulation (ADS) utilizes the principles of Hebbian plasticity to strengthen connections between neurons through the repetitive, reinforced synchronization of neural activity. ADS uses an online system to detect single-unit action potentials in one region to subsequently evoke activity in a different region using intracortical microstimulation (ICMS) pulses. In a model of traumatic brain injury to the caudal forelimb area in the rat (an M1 analogue), ADS was used to reconnect a premotor (rostral forelimb area, RFA) area with primary somatosensory cortex (S1). This treatment was started within several hours of injury and was applied continuously for three weeks, but behavioral improvements were evident within one week and were restored within two. Further, we observed a shift in the neural activity within RFA that was not present with random stimulation (Guggenmos (2013)). To further characterize ADS, we have investigated its ability to rapidly alter firing characteristics in both anesthetized and ambulatory rats without brain injury (Averna, Pasquale et al. 2020, Averna, Hayley et al. 2021). However, due to the nature of cortical injury and the subsequent global disruption in activity and communication, it was unknown what impact ADS would have on the ability to facilitate changes in activity within the trigger and target regions. The purpose of this study is to evaluate $i$ ) the global impact of a focal lesion on the neuronal activity of spared premotor and somatosensory areas and ii) the ability of acute ADS to significantly alter the lesion induced changes. We performed acute experiments in anesthetized rats using a model of focal ischemic lesion in the primary motor area (caudal forelimb area, CFA) using microinjections of a potent vasoconstrictor, i.e. Endothelin-1 (ET-1) (Frost, Barbay et al. 2006). Neurophysiological activity of both the rostral forelimb area (RFA) and the somatosensory cortex (S1) was recorded by using microelectrode arrays. ADS was utilized following the same procedures as previous studies (Averna, Pasquale et al. 2020, Averna, Hayley et al. 2021), by pairing the occurrence of the single-unit activity on a selected channel within RFA with ICMS applied to S1 after CFA ischemic injury.

Understanding the shifts in the neural response following brain injury and coupling this response to our closed-loop stimulation paradigms offers a novel way to probe cortico-cortical circuits and has the potential to inform development of future therapies for acquired brain injury.

\section{Methods \\ Animals}

Nine adult, male Long-Evans rats (weight: 350-400 g, age: 4-5 months; Charles River Laboratories, Wilmington, MA, USA and Charles River Laboratories Italia SRL, Calco, Italy) were utilized in this study. Rats were divided into either the lesioned, no stimulation group (i.e. Control, CTR; $n=3$ ) or the lesioned, stimulation (i.e. Activity-Dependent Stimulation, ADS; $n=6$ ) group (Fig. 1). The experiments were performed both in the USA and in Italy. The University of Kansas Medical Center Institutional Animal Care and Use Committee (USA: protocol 2017-2384 approved on 2/17/17) and the Italian Ministry of Health and Animal Care (Italy: 


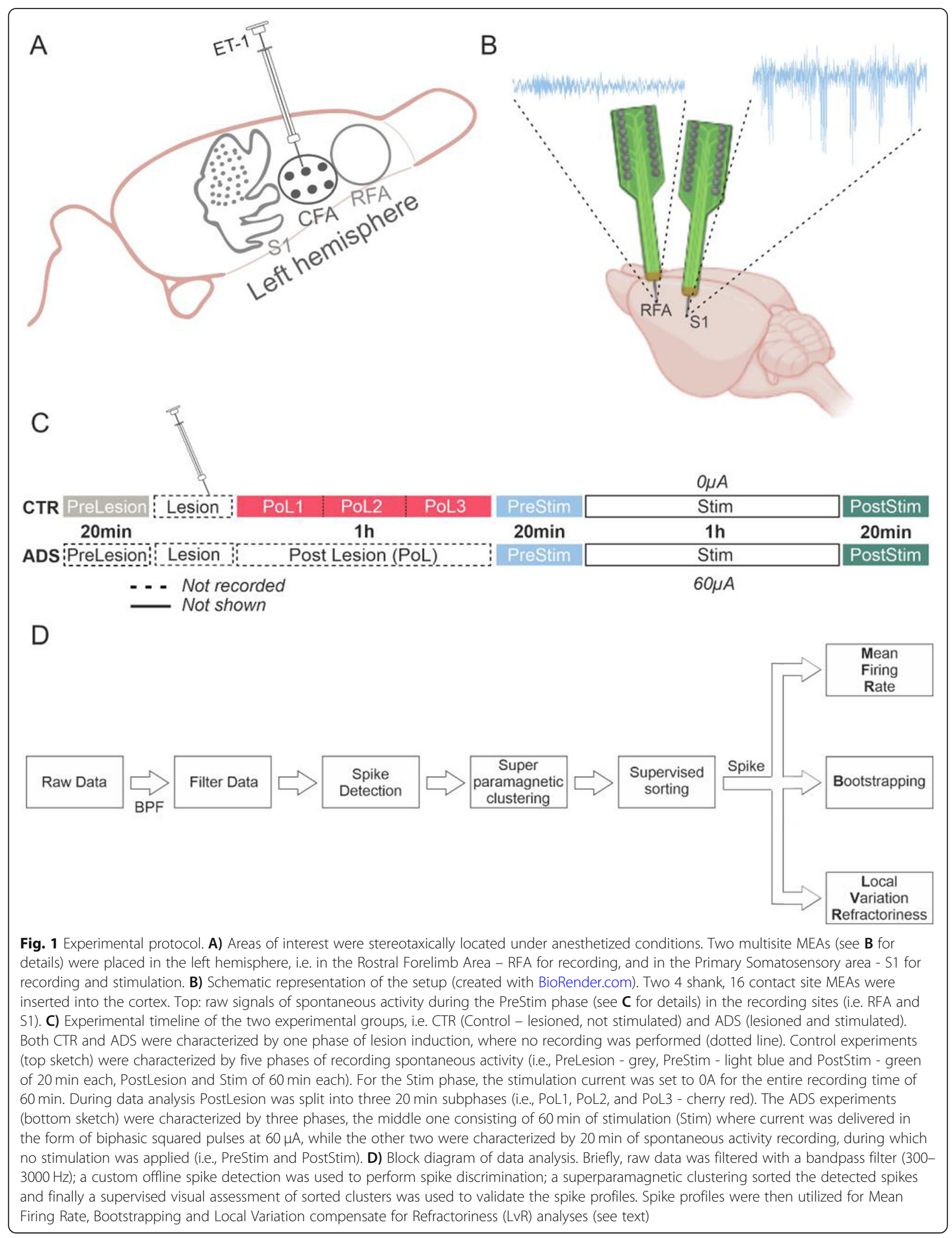


authorization ID 861/2015 PR) approved all experiments performed for this study.

\section{Surgical procedures Preparation}

Rats were assigned to one of the two groups prior to the surgical procedure. Anesthesia was initially performed by placing the rat inside a chamber attached to a vaporizer and introducing gaseous isoflurane (5\%@1 $\mathrm{lpm})$ until induction. A surgical level of anesthesia was obtained by administering ketamine $(80-100 \mathrm{mg} / \mathrm{kg}$ IP) and xylazine $(5-10 \mathrm{mg} / \mathrm{kg})$ and was maintained throughout the entire procedure by bolus injections of ketamine $(10-100 \mathrm{mg} / \mathrm{kg} / \mathrm{hr}$. IM) as needed, whenever a positive pinch or ocular reflex was detected. After securing the rat in the stereotaxic frame, it was placed on a homeostatically controlled heating blanket; temperature and vital parameters were monitored over the duration of the procedure. Lidocaine cream was applied as a topical analgesic prior to performing a midline skin incision spanning rostro-caudally between $\sim 6 \mathrm{~mm}$ rostral to bregma and $\sim 5 \mathrm{~mm}$ distal to the atlanto-occipital junction exposing the skull surface. The muscles of the neck overlying the Cisterna Magna were reflected and a laminectomy was performed in the spinal dura to allow cerebrospinal fluid (CSF) to drain, mitigating brain edema during and after the craniotomy. As shown in Fig. 1A, six $0.65-\mathrm{mm}$ diameter holes were drilled into the skull over the left hemisphere (contralateral to the preferred forelimb) in the area corresponding to the caudal forelimb area (CFA; the M1 forelimb representation analogue in rats) as follows: $+0.5,+2.5 ; 1.5,+2.5 ; 2.5,+$ $2.5 ;+0.5,+3.5 ; 1.5,+3.5 ; 2.5,+3.5 \mathrm{AP}, \mathrm{ML}$ from bregma. Based on standard stereotaxic locations (Kleim, Bruneau et al. 2003), burr holes (3 mm diameter) over RFA and S1 were made at $+3.5,+2.5$ and $-1.25,+4.25 \mathrm{AP}, \mathrm{ML}$, respectively. Once the skull was exposed, the dura mater was removed in these burr holes (RFA and S1) to allow insertion of microelectrode arrays (MEAs; A4x4-5 mm100-125-703-A16, NeuroNexus; Fig. 1B, schematic representation).

\section{Lesion}

An ischemic injury was induced in CFA by intraparenchymal injection of Endothelin-1 (ET-1, Bachem Americas, USA), a potent vasoconstrictor: $0.33 \mu \mathrm{l}$ ET-1 was injected at a rate of $3 \mathrm{nl} / \mathrm{sec}$ via a $160 \mu \mathrm{l}$ pipette (o.d.) attached to a $1 \mu \mathrm{l}$ Hamilton syringe $\sim 1.5 \mathrm{~mm}$ below the pial surface in each of the 6 holes over CFA (Gilmour (2005)). This procedure produced an infarct that encompassed CFA, confined to an area of $0.5 \mathrm{~mm}$ diameter, while leaving RFA and S1 intact (Fang (2010)).

\section{Recording/stimulation paradigms}

Locations for electrode insertions were based on stereotaxic coordinates and blood vasculature patterns. As depicted in Fig. 1B, RFA was implanted with a four-shank, sixteen-contact site electrode with 1-1.5 $\mathrm{M} \Omega$ impedance at each site (A4x4-5 mm-100-125-703-A16, NeuroNexus) at a depth of $1700 \mu \mathrm{m}$. In S1, a similar four-shank, sixteen-contact site electrode was inserted in S1 at a depth of $1200 \mu \mathrm{m}$. The S1 electrode had an activated electrode site within the array, dropping the impedance of this site to roughly $0.2 \mathrm{M} \Omega$ (contact 6 , activated A4x4-5 mm-100703-A16, NeuroNexus) to allow for stimulation. Electrodes were activated by the manufacturer following the procedure described in https://www.neuronexus.com/ files/technicalsupportdocuments/Activation.pdf, and verified by the investigators with impedance testing through the Intan RHS system. Continuous extracellular signals from all channels were amplified, digitized and stored at a sampling rate of $30 \mathrm{kHz}$ using standard, commercially available neurophysiological hardware (Intan RHS, Intan technologies LLC).

For stimulation procedures, a single channel in RFA was selected based on visual observation of spike amplitudes and signal to noise ratios. Once a unit was identified, a channel-specific threshold was set in the Intan software to act as the trigger for activity-dependent stimulation (ADS). Upon each threshold crossing, a single stimulation pulse was delivered to channel 6 on the S1 probe. To prohibit feedback from stimulus-evoked RFA spikes and stimulus artifacts from triggering stimulation, a blanking period $(28 \mathrm{~ms})$ followed each stimulus limiting the maximum stimulation rate to roughly $35 \mathrm{~Hz}$. Each triggered stimulus pulse was a single chargedbalanced biphasic, cathodal-leading pulse $(200 \mu \mathrm{s}$ positive, $200 \mu$ s negative). In the control animals, the pulse current was set at $0 \mu \mathrm{A}$ and for ADS animals this current was set at $60 \mu \mathrm{A}$. All ground pins (for amplification and stimulation) were required to be kept at the same potential. Therefore, all ground pins were bridged and tied to a common point on the animal, either to a skull screw in the intraparietal bone or to a needle embedded in the clavotrapezius muscle.

\section{Experimental protocol}

Experiments were carried out at both the University of Kansas Medical Center (US) and the Istituto Italiano di Tecnologia (Italy) by the same investigators. Due to differences in stereotaxic frames, attachments, and holders, the methodology was modified at the respective locations and was updated as a result. In both settings, there was a difference of roughly $5-10 \mathrm{~min}$ between the insertion of one probe and the insertion of the second probe. Probes were attached to the neurophysiological equipment prior to insertion. Upon insertion, signals were 
monitored to ensure that single-unit activity could be detected; impedance measurements were taken and parameters were determined before data recording began. As described in the 'Animals' section, rats were assigned to one of the two groups: CTR $(n=3)$ and ADS $(n=6)$ which had slightly different experimental protocols as detailed in Fig. 1C. In the CTR group, electrodes were placed in the cortex prior to the lesion induction allowing a period of pre-lesion (i.e., PreLesion - PreL, grey) and post-lesion (i.e., PostLesion - PoL, cherry red) recording phases. In the ADS group, due to technical limitations, the electrodes had to be placed after the induction of the ischemic lesion, limiting pre-lesion data to the CTR group (Fig. $1 \mathrm{C}$, dashed line). In both groups, there was a common 100-min period of recording beginning one hour after the final ET-1 injection which consisted of a 20-min pre-stimulation period (i.e., PreStim PreS, light blue in Fig. $1 \mathrm{C}$ ), a 60-min stimulation period (i.e., Stim, white in Fig. 1 C) and a 20-min poststimulation period (i.e., PostStim - PoS, green in Fig. 1 C).

\section{Data processing}

All data from the two groups was processed in the same way. Data was initially filtered using a 4th order elliptic bandpass filter in the range of $300-3000 \mathrm{~Hz}$ to remove low frequency components within the signal. A custom semi-automatic spike discrimination approach was used to detect and sort spikes from the filtered data. This consisted of a custom offline spike detection algorithm, called Precise Timing Spike Detection (PTSD). PTSD has several parameters that are user-configurable. Peak Lifetime Period (i.e., PLP, sized to contain at most one single spike, set at $2 \mathrm{~ms}$ ) and the Relative Maximum/ Minimum, a peak-to-peak amplitude differential threshold, based on the standard deviation of the noise. This spike detection algorithm ensures that candidate spikes have appropriate parameters to isolate individual units (more details in Maccione, Gandolfo et al. 2009). Spike detection was followed by the freely available superparamagnetic clustering technique developed by the Quiroga group (Quiroga, Nadasdy et al. 2004, Mohammed H 2016) Using a custom Matlab pipeline, spike profiles were validated through a supervised visual assessment of sorted clusters by a single investigator for the entire dataset. This stage is akin to manual "cluster-cutting", in which the user has a visual indicator of selectable spike waveform characteristics (typically the peak-to-peak amplitude). The time frame considered for a spike extends $0.4-\mathrm{ms}$ prior to $0.8-\mathrm{ms}$ after each detected peak (refer to the Matlab code at: https://github.com/m053 m716/CPLtools/tree/master/MatlabAddons/Functions/ Spike\%20Analyses).
Peaks that clearly corresponded to noise were excluded at this stage by assignment to a "noise" or "nonneural" source cluster. All subsequent analyses were performed only on the units identified as neural spikes during the sorting process. For the CTR group, to study the effects of a short-term lesion, we split the 1-h recording session of PostLesion (PoL) into three sequential 20-min sub phases (i.e. PoL1, PoL2 and PoL3 as reported in Fig. $1 C)$.

\section{Mean firing rate}

We evaluated the level of neuronal firing by computing the mean firing rate (MFR, spikes/s) in each experimental phase. We set a minimum firing rate of 0.01 spikes $/ \mathrm{s}$ of all prospective units as the threshold for consideration for analysis (Averna, Hayley et al. 2021).

\section{Bootstrapping method}

For each recording phase of the two groups (i.e., CTR and ADS), we quantified a significant deviation from a null (zero centered) distribution of the differences in firing rates between two time-points for a given unit. Using a bootstrapping method (Slomowitz E 2015, Averna, Pasquale et al. 2020) two time-segments were divided into 1-min bins and then randomly shuffled 10'000 times into two groups. Subsequently, a null-distribution was produced by differences between the means of the two randomly shuffled groups. The real difference falling outside the $95 \%$ confidence interval of the zero distribution was considered significant.

\section{Local variation compensate for refractoriness}

A revised version of the Lv parameter, called Local Variation compensate for Refractoriness (LvR) as proposed in (Shinomoto, Kim et al. 2009), was used to describe the intrinsic firing irregularity of singular neurons. LvR evaluates the local variation of the ISI, assuming that rate dependence is induced by the refractory period of a spike, $R$, which is subtracted from the interspike interval (ISI). The refractoriness constant, $\mathrm{R}$, was set to $5 \mathrm{~ms}$ to intensify the characterization of firing dynamics of individual neurons in terms of $\mathrm{F}$ values (Shinomoto, Kim et al. 2009). The final revised local variation LvR equation is defined as:

$$
L v R=\frac{3}{n-1} \sum_{i=1}^{n-1}\left(1-\frac{4 I_{i} I_{i+1}}{\left(I_{i}+I_{i+1}\right)^{2}}\right)\left(1+\frac{4 \mathrm{R}}{\left(I_{i}+I_{i+1}\right)}\right)
$$

Where $I i$ and $I i+1$ are the $i$-th and $i+1$ st ISIs and $n$ is the number of ISIs. The value provided by this metric, ranged from 0 to more than 2 , is useful to classify the individual neuron's activity into Regular (approx. $0.5 \pm .25$ ), Random (approx. $1 \pm 0.25$ ) and Bursty (approx. $1.5 \pm 0.25$ ) firing patterns (Shinomoto, Kim et al. 2009). 


\section{Statistical analysis}

Statistical analysis was performed in Matlab (The MathWorks, Natick, MA, USA). Data was first tested for normality with the Kolmogorov-Smirnov test. As our data failed the normality test, we used non-parametric tests for our analysis. We performed the Friedman test to detect differences among firing rates at different experimental phases and used the Tukey-Kramer test for posthoc analysis. We used the Wilcoxon signed-rank test to assess statistical differences pre- and post-stimulation within experimental groups, and the Wilcoxon rank-sum test to assess statistical differences among different experimental groups. $P$-values $<0.05$ were considered as significant and corrected for multiple comparisons when relevant.

\section{Results}

An ischemic lesion induced changes of firing activity We evaluated the mean firing rate of RFA and S1 in the CTR group before and after the ischemic lesion. Specifically, we evaluated how the spiking activity of all identified units was impacted by the lesion and how this evolved over time in 20 min increments (Fig. 2). Baseline (Pre Lesion, PreL) activity was characterized by a mix of random spiking and sparse synchronous events across the different electrodes within an MEA in both RFA and $\mathrm{S} 1$, as shown in representative raster plots of one rat (Fig. 2A). After the lesion (i.e., Pre Stim, PreS, about $1 \mathrm{~h}$ after the lesion induction), there was a shift in activity from pre-lesion levels (Fig. 2B). In both the PreL and PreS periods, activity in $\mathrm{S} 1$ was characterized by a higher level of firing than in RFA (4.49 spikes/s in S1 vs. 1.54 spikes/s in RFA PreL; 3.11 spikes/s in S1 vs. 1.8 spikes/s in RFA PreS).

In RFA (Fig. 2C), the lesion induced an overall significant increase in the MFR from the PreL to the PreS period (1.54 spikes/s PreL vs:1.8 spikes/s PreS ${ }^{*} p<$ $5 \cdot 10^{-2}$ Friedman nonparametric test). Significant increases were also observed from the PreL period to PoL1 and from PoL3 to PreS periods (1.54 spikes/s PreL vs: 2.15 spikes/s - PoL1 ${ }^{* * *} p<5 \cdot 10^{-5} ; 1.12$ spikes/s PoL3 vs 1.8 spikes/s PreS, respectively. "** $p<5 \cdot 10^{-5}$ Friedman nonparametric test). Significant decreases in MFR were observed from PreL to PoL3 (1.54 spikes/s PreL vs 1.12 spikes/s PoL3), from PoL1 to PoL2 and PoL3 (2.15 spikes/s PoL1 vs: 1.5 spikes/s PoL2 ${ }^{*} p<$ $5 \cdot 10^{-2} ; 2.15$ spikes/s PoL1 vs 1.12 spikes/s PoL3) and from PoL2 to PoL3 (1.5 spikes/s PoL2 vs 1.12 spikes/s PoL3).

S1 exhibited the opposite trend (Fig. 2D), characterized by a significant decrease in the MFR from the PreL to the PreS period (4.49 spikes/s PreL vs 3.11 spikes/s PreS, ${ }^{* * *} p<5 \cdot 10^{-5}$ Friedman non parametric test). This decreasing trend was observed for all PoL periods with respect to PreL (4.49 spikes/s PreL vs 3.04 spikes/s PoL1, 2.52 spikes/s PoL2, 1.97 spikes/s PoL3, ${ }^{* * *} p<5 \cdot 10^{-5}$ Friedman non parametric test) and from PoL1 and PoL2 to PoL3 (3.04 spikes/s PoL1, 2.52 spikes/s PoL2 vs: 1.97 spikes/s PoL3, *** $p<5 \cdot 10^{-5}$ Friedman non parametric test). There was an observed recovery of firing rate between PoL3 and PreS (1.97 spikes/s PoL3 vs 3.11 spikes/s PreS, *** $p<5 \cdot 10^{-5}$ Friedman non parametric test).

\section{An ischemic lesion affected the number of firing units}

To compare single unit firing activity in the five experimental phases (i.e., PreL, PoL1, PoL2, PoL3 and PreS) and identify possible changes, we evaluated whether the difference in values between two considered phases (i.e., PreL vs. PoL1-3 and PreL vs PreS) for a given unit significantly deviated from a null (zero centered) distribution using a bootstrapping method (see the 'Data Processing - Bootstrapping Method section in the Materials and Methods), as reported in Fig. 3A. We calculated the fraction of units whose firing significantly increased, decreased or remained constant (i.e., according to the bootstrapping method) between i) the Pre Lesion and each of the PoL1, PoL2 and PoL3 phases (Fig. 3A1, panels from left to right) in RFA, and ii) the Pre Lesion and each of the PoL1, PoL2 and PoL3 phases (Fig. 3A2, panels from left to right) in S1.

In case $i$ ), during the immediate Post Lesion phase, about $52 \%$ of the units showed an increase in the firing rate. This percentage decreased across the entire Post Lesion condition reaching as low as $30 \%$, returning to values around 50\% during Pre Stim (Fig. 3B1). The number of units that decreased their firing with respect to the baseline constantly increased during the Post Lesion phase, up to $44 \%$, with a decrease in the PreS, where the percentage reached values around $35 \%$. The number of units that showed no change was stable for the entire PoL1-3 and PreS phases, around $16-25 \%$.

In case ii), during the immediate Post Lesion phase (i.e. PoL1), about $55 \%$ of the units show an increase in the firing rate. This percentage decreased during both PoL2 and, in a greater way, PoL3, reaching the minimum value of 34\% (Fig. 3B2). In PreS, we observed a jump in the number of increased units (i.e. 57\%), with values even higher than the Pre Lesion condition. The number of units that decreased their firing with respect to the baseline slightly increased during the Post Lesion phase but returned to values comparable to the Pre Lesion during PreS condition. As in RFA, the number of units that showed no change was stable for the entire PoL1-3 and PreS phases, with values in the range $8-25 \%$.

\section{ADS increased the global level of firing}

We evaluated the level of firing of RFA and S1 in both the ADS and CTR group, before (i.e., Pre Stimulation, 


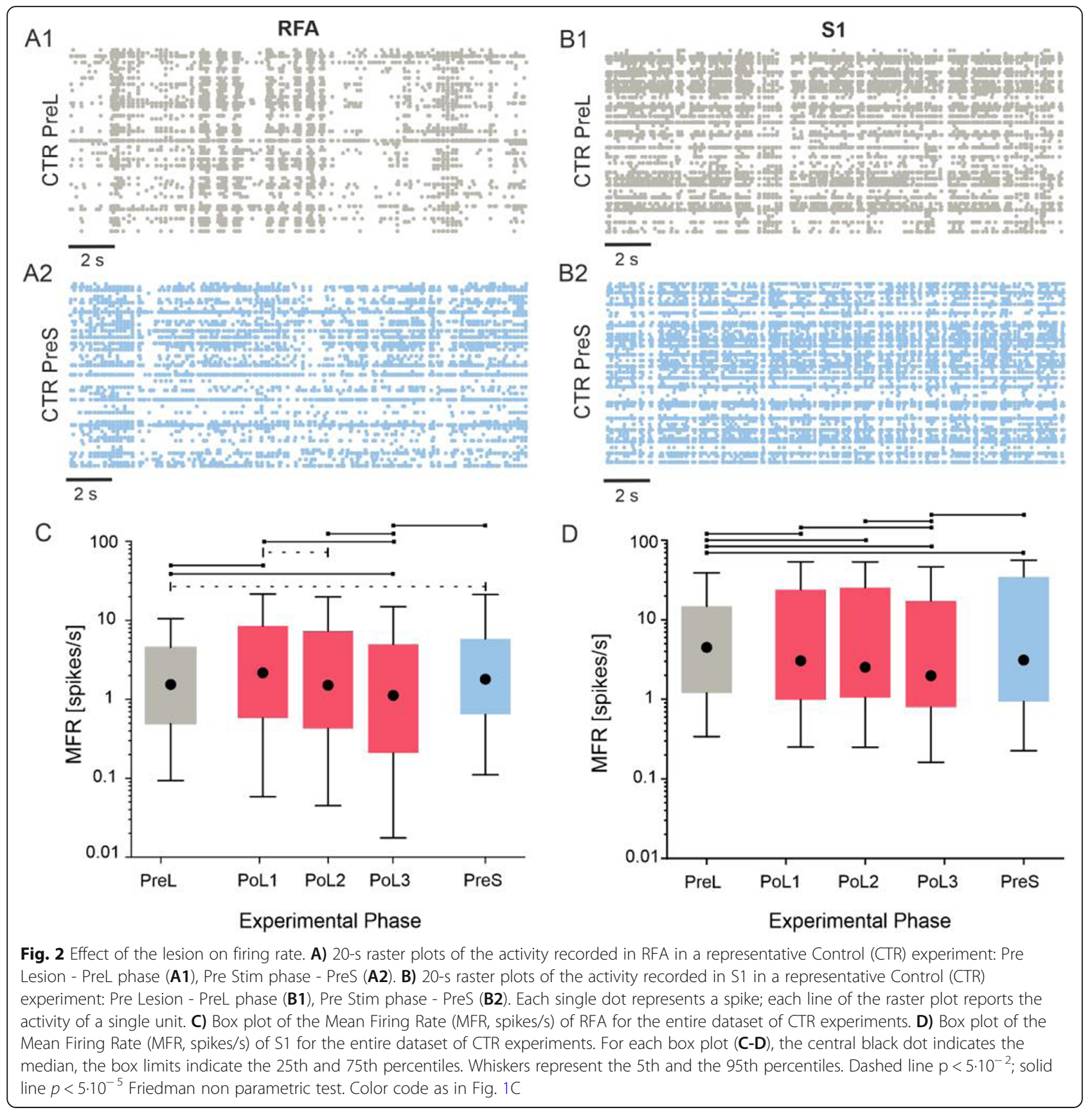

PreS) and after the stimulation (i.e. Post Stimulation, PoS) phase (Fig. 4). In the ADS group in RFA, the baseline activity PreS was characterized by random spiking with some synchronous events, as shown in the raster plot of one representative ADS experiment (Fig. 4A1). After the closed-loop stimulation, in the PoS phase, we observed a global increase of activity, with massive network-wide synchronous events (Fig. 4A2) which was not observed in the PoS of the Control group (CTR), as shown by a CTR representative raster plot (Fig. 4B), where a sustained level of firing is exhibited, but no collective, synchronous patterns are present. No difference was found in the CTR group (1.8 spikes/s PreS vs 2.1 spikes/s PoS: n.s., Wilcoxon signed rank test), while the ADS exhibited a significant increase in the level of firing after the stimulation session (0.96 spikes/s PreS vs 1.48 spikes/s PoS: ${ }^{*} p<8 \cdot 10^{-3}$, Wilcoxon signed rank test). The ratio between PoS and PreS (Fig. 4C) highlighted the significant increase induced by the stimulation in the ADS group with respect to CTR. This indicates that the closed loop stimulation was able to significantly increase the global level of firing in RFA. 


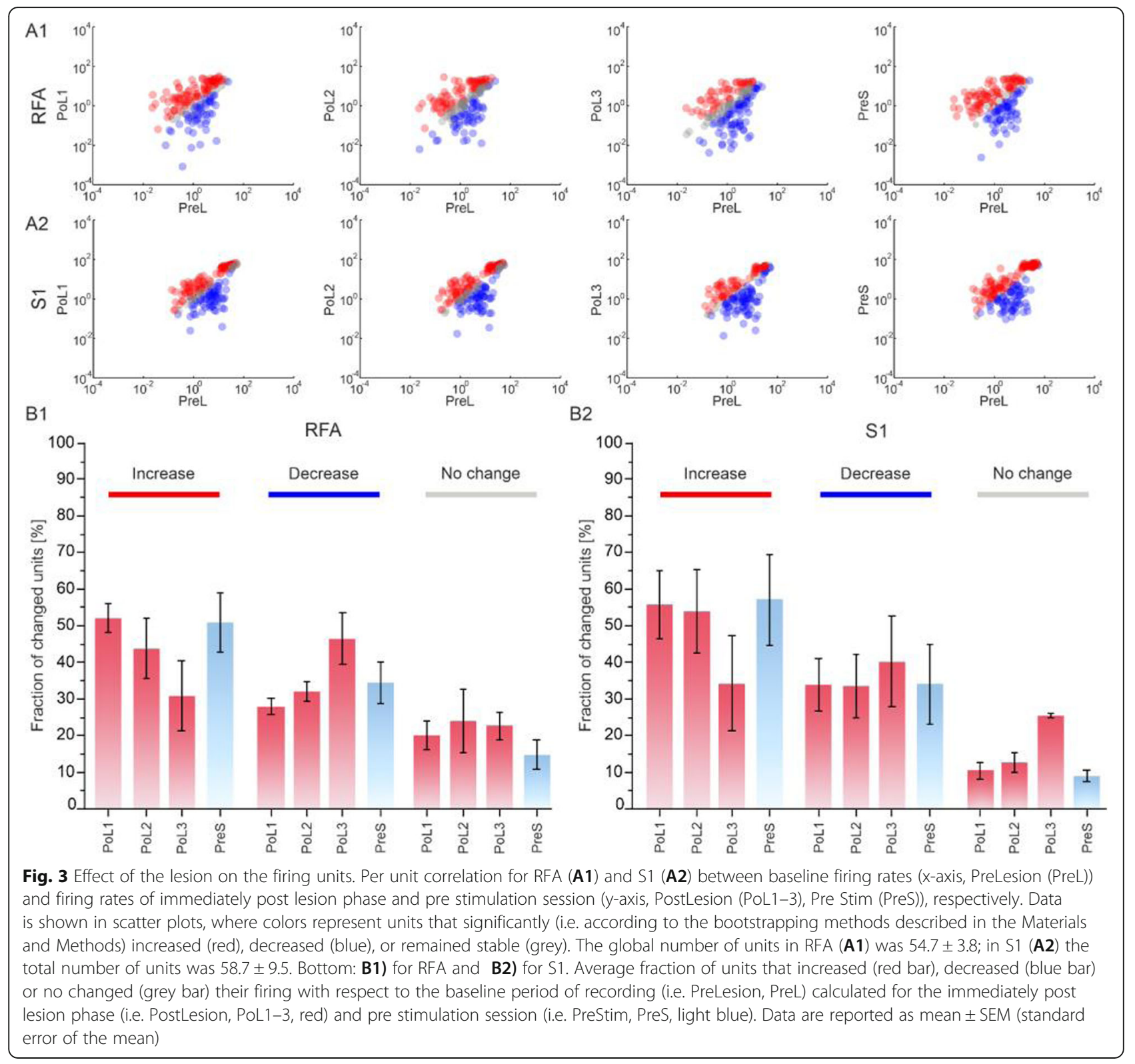

In both groups in S1, a similar trend to RFA was observed. The raster plot of a representative ADS experiment is shown in Fig. 4D. Similar spiking characteristics to RFA were observed in S1 (Fig. 4D1). After the stimulation, there was also a general increase of activity characterized by network-wide bursting (Fig. 4A2). In the CTR experiment, after the $0 \mu \mathrm{A}$ stimulation, the level of firing is higher than in ADS (Fig. 4F) but characterized by less synchronicity. The PreS and PoS conditions for both CTR and ADS had a statistically significant increase in activity both in CTR and ADS (3.11 spikes/s PreS vs 4.43 spikes/s PoS for CTR and 0.54 spikes/s PreS vs 0.64 spikes/s $\operatorname{PoS}$ for $\mathrm{ADS}:{ }^{* *} p<8 \cdot 10^{-5}$ Wilcoxon signed rank test).

\section{ADS affected the number of firing units}

To compare single unit firing activity before and after the stimulation and evaluate possible changes, the difference in values between the two phases (i.e., PoS vs PreS) for a given unit significantly deviated from a null (zero centered) distribution using a bootstrapping method (see the 'Data Processing - Bootstrapping Method section in the Materials and Methods), as reported in Fig. 5A, B. The fraction of units whose firing significantly increased, decreased or remained constant (i.e. according to the bootstrapping method) in RFA (Fig. 5A) and in S1 (Fig. $5 \mathrm{~B})$ was calculated. The quantification of the previous results is reported in Fig. 5C for RFA and Fig. 5D for S1. In Fig. 5C (RFA), after the stimulation, about $28 \%$ of the 


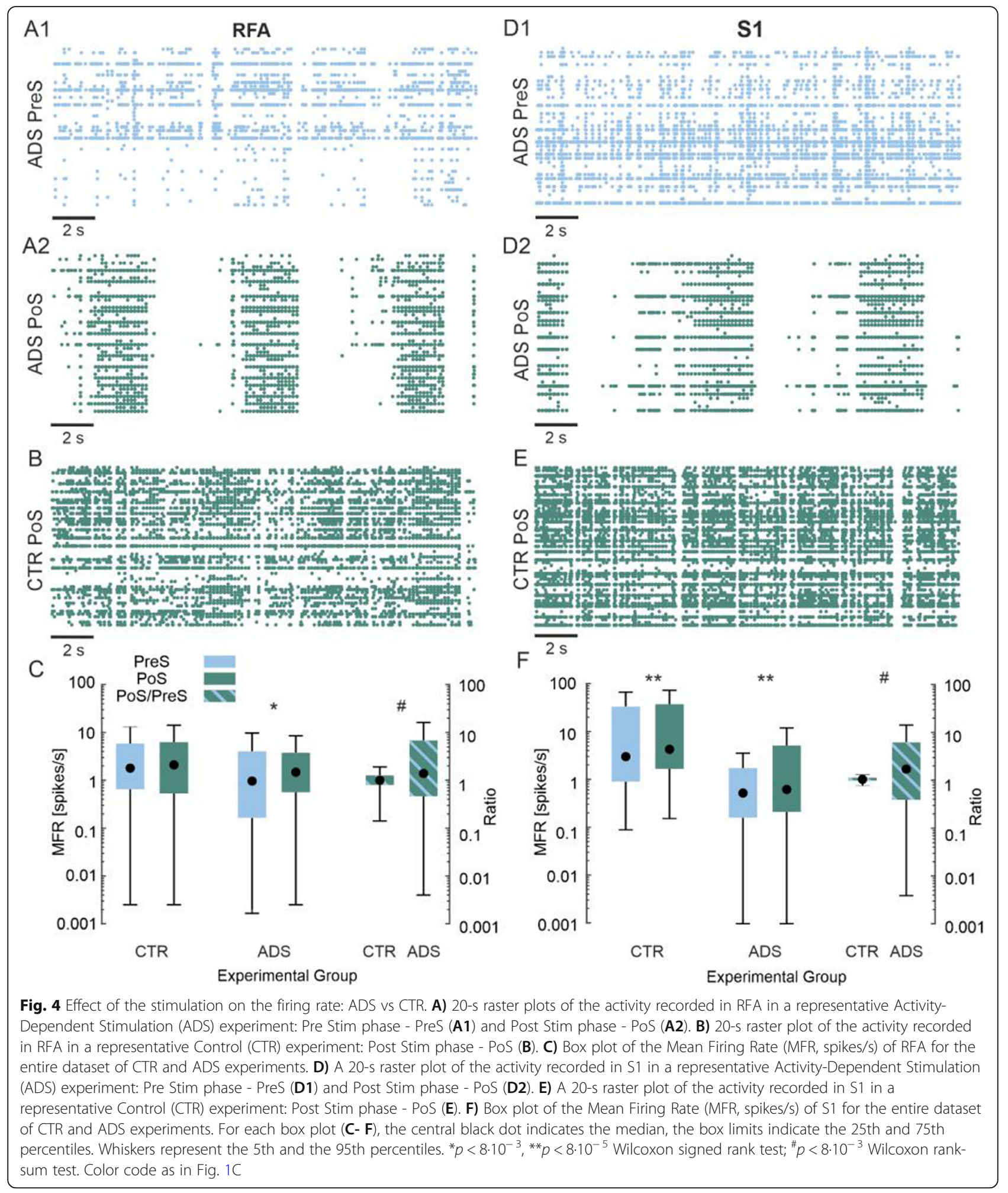

units in CTR and $38 \%$ of the units in ADS show an increase in the firing rate. The percentage of decreased units in PoS vs PreS is around $25 \%$ for both CTR and ADS. The number of units that showed no change is around $45 \%$ in CTR and 35\% in ADS, but with ADS exhibiting higher variability. In Fig. 5D (S1), the number of increased units is around 58\% in ADS while is $48 \%$ in CTR, but highly variable. In S1, the number of decreased 


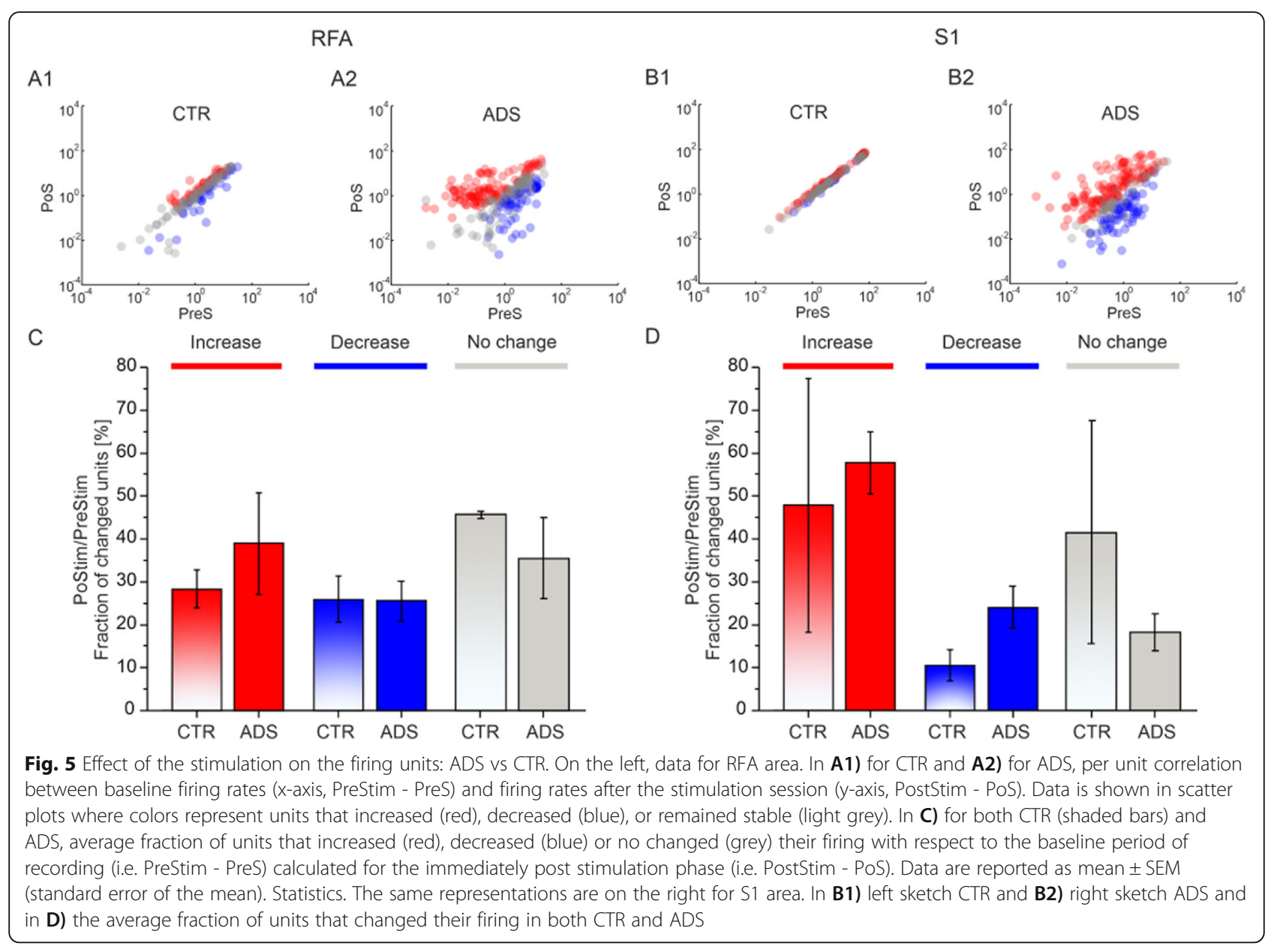

units is higher in ADS (24\%) than in CTR (10\%), while the number of no change units is high and very variable in the CTR (41\%) with respect to the ADS (18\%) group.

\section{ADS induced a shift from random firing to bursting activity}

To further investigate possible changes in the firing patterns induced by either the lesion or the stimulation, we classified the type of spike-firing pattern of recorded neurons in both RFA and S1 through the LvR coefficient. As reported in Fig. 6, neurons of both RFA and S1 exhibited stable baseline firing patterns Pre Lesion, with values defining a "Random" state (cf. Figure 6A: $\mathrm{LvR}=$ $1.21 \pm 0.25$ and Fig. 6B: $\operatorname{LvR}=1.12 \pm 0.19$, mean \pm SD). After the lesion, the level of LvR, measured both within RFA and S1 was constant for the entire duration of the experiment (cf. Figure 6C, D). The effects of stimulation on LvR are reported in Fig. 6E and F. As demonstrated in the histograms of Fig. 6E1, CTR experiments showed an overlap of the distribution obtained during the PreS and PoS conditions. In the ADS experiments, there was a shift of the distributions towards the right indicating a global increase of LvR values in RFA, moving the patterns of activity from the "Random" condition of the PreS to the "Bursty" state of firing in PoS (Fig. 6E2). The quantification of this change was obtained by comparing the LvR PreS/PoS ratio between the CTR and ADS groups (Fig. 6E3). We found a statistically significant increase of ADS with respect to CTR (ADS vs CTR: ** $p<$ $5 \cdot 10^{-3}$ Wilcoxon rank-sum test). In S1 we obtained results similar to those obtained in RFA. The histograms show a strong overlap in the PreS and PoS distributions of LvR for CTR experiments while the distribution shifted towards higher LvR values in the ADS group (Fig. 6F1 and 6F2 respectively). By comparing the LvR PreS/PoS ratio between the CTR and ADS groups (Fig. 6F3), we found a statistical increase of ADS with respect to CTR (ADS vs CTR: *** $p<5 \cdot 10^{-3}$ Wilcoxon rank-sum test).

\section{Discussion}

The purpose of this study was to investigate alterations in single-unit firing properties of neurons within ipsilesional spared sensorimotor areas immediately following an ischemic injury to M1. During an ischemic event, neurons within the penumbra can display altered firing 


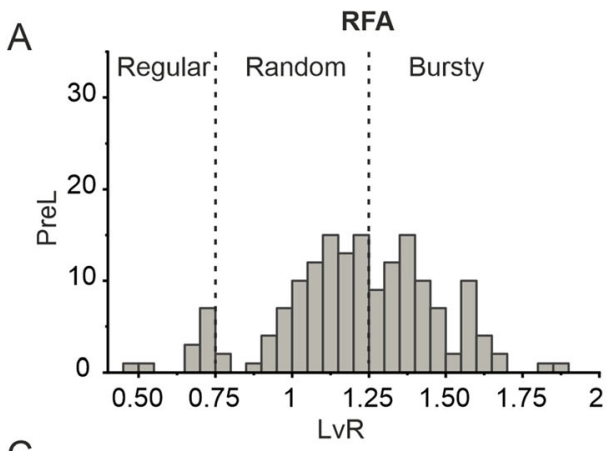

C

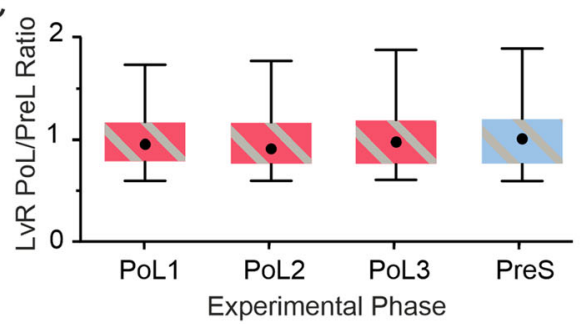

E1

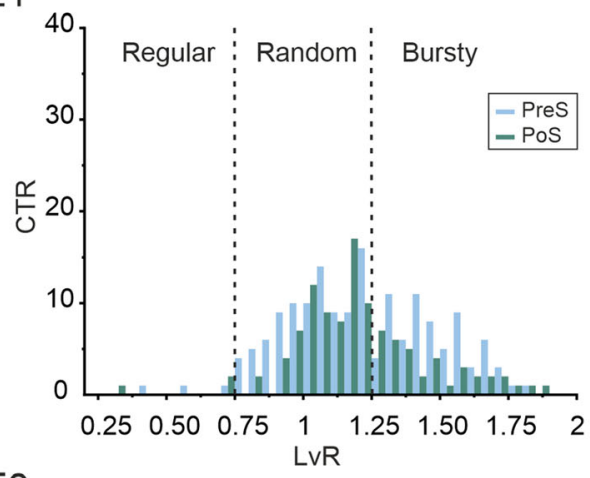

E2
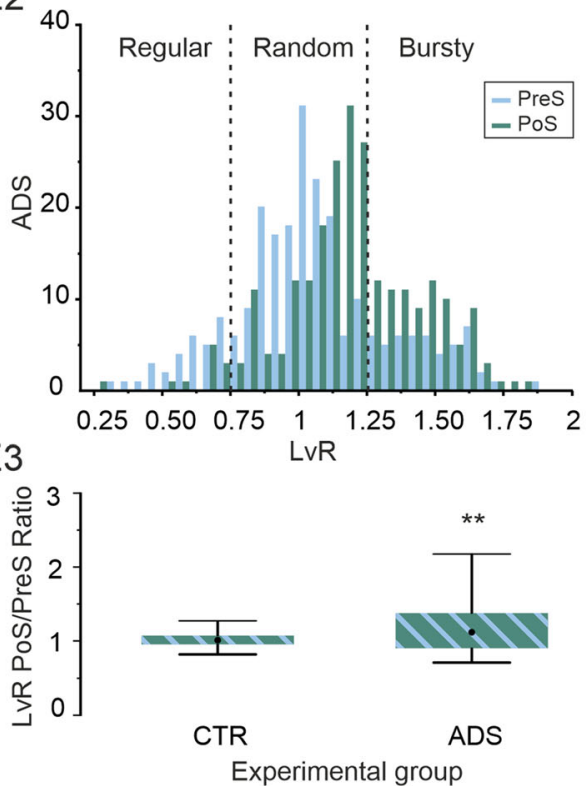

B

S1

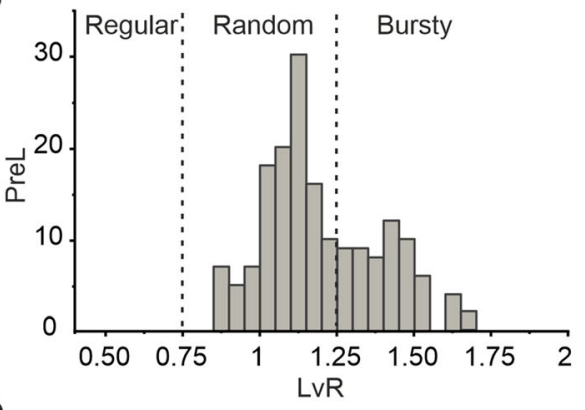

D

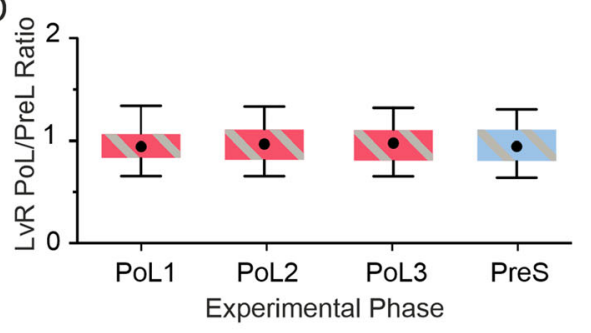

F1

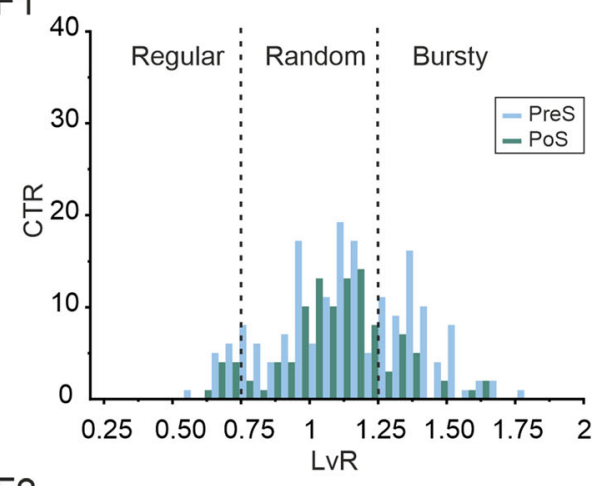

F2
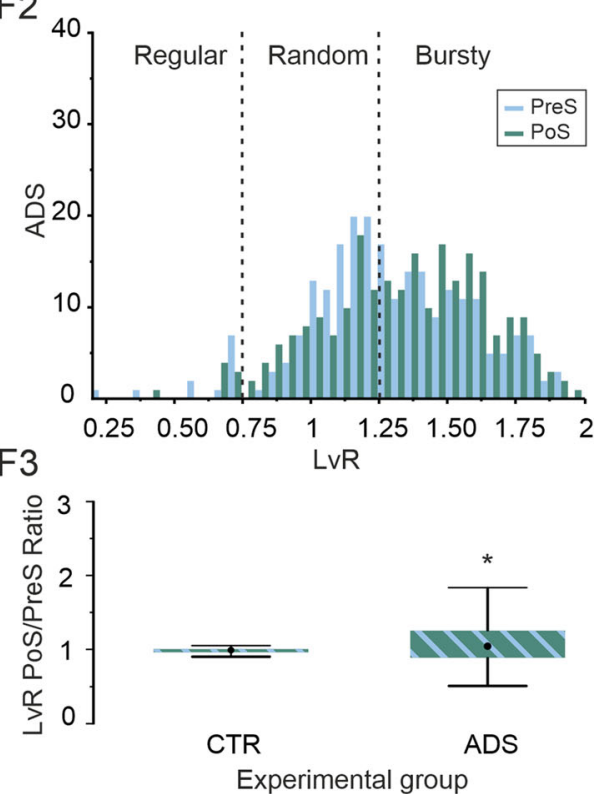

Fig. 6 (See legend on next page.) 


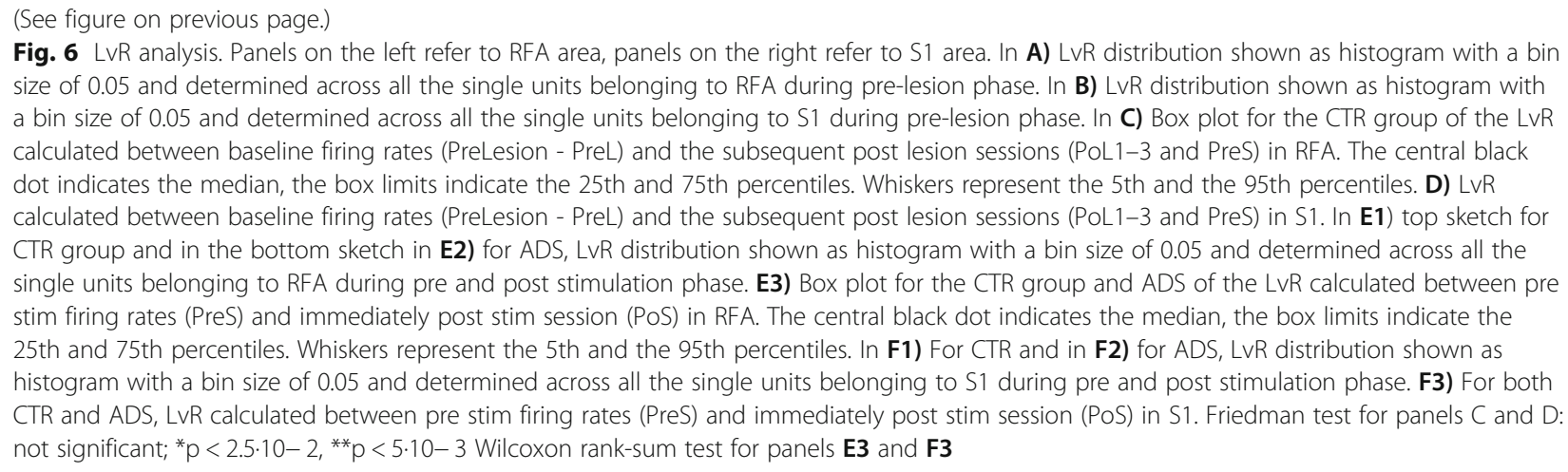

patterns, with a cessation of activity as blood flow is decreased (Heiss, Hayakawa et al. 1976). While the likelihood of neuronal survival in the ischemic core is dependent on reperfusion of blood flow, any significant alteration in activity will likely cause a subsequent disruption in the firing patterns of neurons that were connected to the affected region (Bauer, Kraft et al. 2014). Further, it is well established that ischemic injury can lead to an increase in glutamatergic release in the penumbra which can lead to an increase in activity, which can then feedback into an excitotoxic response in the hours immediately after injury (Rothman and Olney 1986). ET-1 injected into CFA induced a permanent ischemic injury, which led to rapid cell death in the core. Because CFA has strong, reciprocal projections to ipsilateral somatosensory and motor areas, the rapid loss of any inhibitory outputs to these areas, combined with any lesion-related glutamatergic response would suggest that an increase in the activity in both of these regions should be observed. Interestingly, RFA showed an increase of the global mean firing rate in the periods immediately after injury while S1 showed a consistent decrease over the same periods. There are a number of potential explanations for the variability in response. First, these areas are some distance from the ischemic core and are likely to have a more complex and variable response to an array directly in the penumbra. Second, the methodology for inducing the lesion across M1 takes a minimum of roughly $30 \mathrm{~min}$ to complete across the six injection locations. Therefore there is likely to be some timing differences between when more rostral or caudal areas of M1 are impacted, leading to potentially different results in PM and S1. Further analysis on individual units indicated that over half of the identified units increased their firing rate over the post-lesion period in both RFA and S1 even as the overall mean firing rate changed. This may be the result of neuronal sub-types having differential responses to the injury.

ADS is a potent way to synchronize the output of one neuron with the forced, evoked activity (through electrical stimulation) of another population of neurons. This was first demonstrated by (Jackson, Mavoori et al. 2006), to alter motor output within M1 of the macaque, and was further utilized by Guggenmos (2013), as a way to facilitate recovery after M1 injury in the rat by coupling premotor and somatosensory areas. While behavioral demonstrations offer the most direct indication of the efficacy of this approach, the underlying mechanisms, while based on spike-timing dependent plasticity, are still under investigation. We have previously described the impact of ADS on firing patterns both in anesthetized and ambulatory brain-intact rats. In the normal anesthetized case, ADS induces an increase in the overall MFR while decreasing the observed LvR in RFA, indicating that triggered stimulation in S1 was able to alter activity patterns in RFA even after stimulation ceased. Here, we observed a similar response in MFR in both RFA and S1 while LvR increased (or became more bursty) in RFA.

This trend in LvR to become more bursty is likely due to the observed shift in activity of the ADS (but not CTR) to induce a cyclic pattern of activity in the sub $1 \mathrm{~Hz}$ range. The majority of ADS animals showed this cyclic pattern of activity, but it is unclear what is underpinning these bursts. If it were merely a carryover effect from the ADS, the stimulation frequency and subsequent entrainment should have been at higher $(2-10 \mathrm{~Hz})$ frequencies. (Carmichael and Chesselet 2002) described these low frequency waveforms with similar subsequent bursting of activity in perilesional cortex at one day post-injury with a thermocoagulatory lesion to CFA. These low-frequency waveforms are thought to synchronize neural activity that would ultimately shape neuroplastic mechanisms such as axonal sprouting. Our data suggests that ADS may be able to induce these patterns of synchronous activity rapidly after injury. Further investigation in both stimulated and non-stimulated animals is needed to assess the role stimulation may have in promoting and shaping these effects. 


\section{Conclusion}

In this study, we performed acute experiments in anesthetized rats affected by a focal lesion in the motor area. We recorded the electrophysiological activity of both premotor (RFA) and somatosensory (S1) cortex, in order to evaluate (i) how the focal lesion affects the activity of the two areas and (ii) whether acute closed-loop intracortical microstimulation (i.e. Activity-Dependent Stimulation, ADS) can alter the lesion induced changes. We first evaluated the immediate, global impact on neural activity of the focal lesion. We found that the ischemic injury resulted in an overall increase in the MFR within RFA but a decrease in S1, while simultaneously observing that the largest proportion of individual neurons increased their firing rates. Stimulation induced a further increase in the global MFR and the proportion of individual units increasing their firing. Stimulation also resulted in a shift in the pattern of activity from random to more bursty. Coupled with the observation of low-frequency synchronous activity within MEAs, it is clear that ADS can rapidly alter the intrinsic neural activity after a focal lesion. This work contributes to our growing understanding of how stimulation paradigms alter damaged brain networks and represents a fundamental step for the development of future therapies for acquired brain injury.

\section{Abbreviations}

M1: Primary Motor cortex; ADS: Activity-Dependent Stimulation; MEA: Microelectrode Array; RFA: Rostral Forelimb Area; S1: Somatosensory cortex; TBI: Traumatic Brain Injury; CFA: Caudal Forelimb Area; ET1: Endothelin-1; CTR: Control group; CSF: Cerebrospinal Fluid; PTSD: Precise Timing Spike Detection; MFR: Mean Firing Rate; LVR: Local Variation compensate for Refractoriness.

\section{Acknowledgements}

The authors would like to thank Dr. Fellin and his group at IIT for hosting part of our experiments in his Labs.

\section{Authors' contributions}

A.A., R.J.N., M.C., and D.G. designed the study. D.G. and M.C. coordinated the study. A.A. and D.G. performed the experiments. A.A., M.Ca., F.B., M.S., and M.C. designed the computational methods. M.Ca. performed the sorting procedures. A.A., M.Ca., and F.B., performed the analysis. M.Ca., F.B., and M.S. performed the statistical analysis, M.Ca., F.B. and M.C. and prepared the Figs. M. Ca, A.A., M.C. and D.G. wrote the first draft of the manuscript. All the authors contributed to and revised the manuscript. R.J.N., D.G., L.D.M., and M.C. collected the funding for the study. All the authors have read and approved the manuscript.

\section{Funding}

Research supported by Italian Ministry of Foreign Affairs and International Collaboration (MAECI), Directorate General for Country Promotion, as a highrelevance bilateral project within the Italy-USA; National Institutes of Health (grants R01NS030853, R03HD094608).

\section{Availability of data and materials}

Data collected and analyzed from this study are available on reasonable request.

\section{Declarations}

Ethics approval and consent to participate

The University of Kansas Medical Center Institutional Animal Care and Use Committee (USA: protocol 2017-2384 approved on 2/17/17) and the Italian Ministry of Health and Animal Care (Italy: authorization ID 861/2015 PR) approved all experiments.

\section{Consent for publication}

Not applicable.

\section{Competing interests}

The authors declare that they have no known competing financial interests or personal relationships that could have appeared to influence the work reported in this paper.

\section{Author details}

${ }^{1}$ Rehab Technologies, Istituto Italiano di Tecnologia, 16163 Genoa, Italy. ${ }^{2}$ Department of Informatics, Bioengineering, Robotics System Engineering (DIBRIS), University of Genova, 16145, Genoa, Italy. ${ }^{3}$ Aldo Ravelli Research Center for Neurotechnology and Experimental Neurotherapeutics, Department of Health Sciences, University of Milan, 20142 Milan, Italy. ${ }^{4}$ Department of Rehabilitation Medicine, University of Kansas Medical Center, Kansas City 66160, USA. ${ }^{5}$ Landon Center on Aging, University of Kansas Medical Center, Kansas 66160, USA.

Received: 24 November 2021 Accepted: 27 January 2022

Published online: 28 February 2022

\section{References}

Averna A, Hayley P, Murphy MD, Barban F, Nguyen J, Buccelli S, et al. Entrainment of network activity by closed-loop microstimulation in healthy ambulatory rats. Cereb Cortex. 2021;31(11):5042-55. https://doi.org/10.1093/ cercor/bhab140.

Averna A, Pasquale V, Murphy MD, Rogantin MP, Van Acker GM, Nudo RJ, et al. Differential effects of open- and closed-loop Intracortical microstimulation on firing patterns of neurons in distant cortical areas. Cereb Cortex. 2020;30(5): 2879-96. https://doi.org/10.1093/cercor/bhz281.

Bauer AQ, Kraft AW, Wright PW, Snyder AZ, Lee JM, Culver JP. Optical imaging of disrupted functional connectivity following ischemic stroke in mice. Neuroimage. 2014;99:388-401. https://doi.org/10.1016/j.neuroimage.2014 05.051 .

Belagaje SR. Stroke rehabilitation. Contin (Minneap Minn). 2017;23(1, cerebrovascular disease):238-53.

Carmichael ST, Chesselet M-F. Synchronous neuronal activity is a signal for axonal sprouting after cortical lesions in the adult. J Neurosci. 2002;22(14):6062-70. https://doi.org/10.1523/JNEUROSCI.22-14-06062.2002.

Coleman ER, Moudgal R, Lang K, Hyacinth HI, Awosika OO, Kissela BM, et al. Early rehabilitation after stroke: a narrative review. Curr Atheroscler Rep. 2017; 19(12):59. https://doi.org/10.1007/s11883-017-0686-6.

Dimyan MA, Cohen LG. Neuroplasticity in the context of motor rehabilitation after stroke. Nat Rev Neurol. 2011;7(2):76-85. https://doi.org/10.1038/ nrneurol.2010.200.

Fang P, Barbay S, Plautz EJ, Hoover E, Strittmatter SM, Nudo RJ. Combination of NEP 1-40 treatment and motor training enhances behavioral recovery after a focal cortical infarct in rats. Stroke. 2010;41(3):544-9.

Frost SB, Barbay S, Mumert ML, Stowe AM, Nudo RJ. An animal model of capsular infarct: endothelin-1 injections in the rat. Behav Brain Res. 2006;169(2):20611. https://doi.org/10.1016/j.bbr.2006.01.014.

Gilmour G, Iversen SD, O'Neill MF, O'Neill MJ, Ward MA, Bannerman DM. Amphetamine promotes task-dependent recovery following focal cortical ischaemic lesions in the rat. Behav Brain Res. 2005;165:98-109.

Guggenmos DJ, Azin M, Barbay S, Mahnken JD, Dunham C, Mohseni P, et al. Restoration of function after brain damage using a neural prosthesis. Proc Natl Acad Sci. 2013;110:21177-82.

Heiss WD, Hayakawa T, Waltz AG. Cortical neuronal function during ischemia. Effects of occlusion of one middle cerebral artery on single-unit activity in cats. Arch Neurol. 1976;33(12):813-20. https://doi.org/10.1001/archneur.1976. 00500120017003 
Jackson A, Mavoori J, Fetz EE. Long-term motor cortex plasticity induced by an electronic neural implant. Nature. 2006;444(7115):56-60. https://doi.org/10.1 038/nature05226.

Kleim JA, Bruneau R, VandenBerg P, MacDonald E, Mulrooney R, Pocock D. Motor cortex stimulation enhances motor recovery and reduces peri-infarct dysfunction following ischemic insult. Neurol Res. 2003;25(8):789-93. https:// doi.org/10.1179/016164103771953862.

Lang CE, Waddell KJ, Barth J, Holleran CL, Strube MJ, Bland MD. Upper limb performance in daily life approaches plateau around three to six weeks poststroke. Neurorehabil Neural Repair. 2021;35(10):903-14. https://doi.org/10.11 77/15459683211041302.

Maccione A, Gandolfo M, Massobrio P, Novellino A, Martinoia S, Chiappalone M. A novel algorithm for precise identification of spikes in extracellularly recorded neuronal signals. J Neurosci Methods. 2009;177(1):241-9. https:// doi.org/10.1016/j.jneumeth.2008.09.026.

Mang CS, Campbell KL, Ross CJ, Boyd LA. Promoting neuroplasticity for motor rehabilitation after stroke: considering the effects of aerobic exercise and genetic variation on brain-derived neurotrophic factor. Phys Ther. 2013; 93(12):1707-16. https://doi.org/10.2522/ptj.20130053.

Mohammed H, J. N. Ipsilateral cortical inputs to the rostral and caudal motor areas in rats. J Comp Neurol. 2016;524(15):3104-23.

Murphy TH, Corbett D. Plasticity during stroke recovery: from synapse to behaviour. Nat Rev Neurosci. 2009;10(12):861-72. https://doi.org/10.1038/nrn2 735.

Organization, W. H. (2018). "WHO - the top 10 causes of death.".

Phipps MS, Cronin CA. Management of acute ischemic stroke. Bmj. 2020;368: 16983.

Quiroga RQ, Nadasdy Z, Ben-Shaul Y. Unsupervised spike detection and sorting with wavelets and superparamagnetic clustering. Neural Comput. 2004;16(8): 1661-87. https://doi.org/10.1162/089976604774201631.

Rothman SM, Olney JW. Glutamate and the pathophysiology of hypoxicischemic brain damage. Ann Neurol. 1986;19(2):105-11. https://doi.org/10.1 002/ana.410190202

Semprini M, Laffranchi M, Sanguineti V, Avanzino L, De Icco R, De Michieli L, et al. Technological approaches for neurorehabilitation: from robotic devices to brain stimulation and beyond. Front Neurol. 2018;9(212). https://doi.org/10.33 89/fneur.2018.00212.

Shinomoto S, Kim H, Shimokawa T, Matsuno N, Funahashi S, Shima K, et al. Relating neuronal firing patterns to functional differentiation of cerebral cortex. PLoS Comput Biol. 2009;5(7):e1000433. https://doi.org/10.1371/journal. pcbi.1000433.

Slomowitz ESB, Vertkin I, Milshtein-Parush H, Nelken I, Slutsky M, et al. Interplay between population firing stability and single neuron dynamics in hippocampal networks. Elife. 2015;4. https://doi.org/10.7554/eLife.04378.

\section{Publisher's Note}

Springer Nature remains neutral with regard to jurisdictional claims in published maps and institutional affiliations.

Ready to submit your research? Choose BMC and benefit from:

- fast, convenient online submission

- thorough peer review by experienced researchers in your field

- rapid publication on acceptance

- support for research data, including large and complex data types

- gold Open Access which fosters wider collaboration and increased citations

- maximum visibility for your research: over $100 \mathrm{M}$ website views per year

At BMC, research is always in progress.

Learn more biomedcentral.com/submissions 